\title{
Desafios para a sustentabilidade dos periódicos científicos brasileiros e do Programa SciELO
}

\author{
Challenges for the sustainability of Brazilian scientific journals \\ and the SciELO Program
}

Leila Posenato Garcia (https://orcid.org/0000-0003-1146-2641) ${ }^{1}$

Antonio Fernando Boing (https://orcid.org/0000-0001-9331-1550) ${ }^{2}$

${ }^{1}$ Fundação Jorge Duprat e Figueiredo de Segurança e Medicina do Trabalho. R. Silva Jardim 213, Centro. 88020-200 Florianópolis SC Brasil.leila.garcia@ fundacentro.gov.br ${ }^{2}$ Departamento de Saúde Pública, Universidade Federal de Santa Catarina. Florianópolis SC Brasil.

\begin{abstract}
Scientific journals play a fundamental role in the development of science. However, in Brazil, there are major challenges for their sustainability, as well as for the sustainability of the SciELO Program. The article reports a worrying scenario of low public funding and lack of strategic planning for long-term funding for scientific publication, contrary to the development of Brazilian journals and the SciELO Program. This scenario places great pressure on journals, with reports of situations of closure of activities, or implementation of fees charged to authors, while bringing financial insecurity to the SciELO Program. The actions taken by Brazilian scientific societies and the position of the Forum of Editors of Public Health Journals are aligned with that of the Advisory Committee of the SciELO Brazil Collection, namely that the publication of scientific journals edited in Brazil is strategic and, therefore, should be fostered through public policies and supported with public financing from the sponsoring institutions and grants from the research funding institutions.
\end{abstract}

Key words Policies of support and financing of scientific journals, Expenditure indicators on Science and Technology, National policy on Science, Technology and Innovation
Resumo As revistas científicas têm papel fundamental para o desenvolvimento da ciência. Contudo, no Brasil, existem grandes desafios para sua sustentabilidade, assim como do Programa SciELO. O artigo relata um cenário preocupante de baixo financiamento público e ausência de planejamento estratégico com financiamento de longo prazo para a publicação científica, na contramão do desenvolvimento das revistas brasileiras e do Programa SciELO. Tal cenário impõe grande pressão às revistas, com relatos de situações de encerramento de atividades, ou implementação de cobrança de taxas aos autores, ao mesmo tempo que traz insegurança financeira ao Programa SciELO. São informadas as ações tomadas por sociedades científicas Brasileiras e a posição do Fórum de Editores das Revistas da Saúde Coletiva, alinhada à do Comitê Consultivo da Coleção SciELO Brasil, de que a publicação de periódicos científicos editados no Brasil é estratégica e, portanto, deve ser incentivada por meio de politicas públicas e apoiada com recursos públicos provenientes das instituições mantenedoras e dos editais das agências de fomento.

Palavras-chave Politicas de apoio e financiamento da edição de publicações científicas, Indicadores de gastos em Ciência e Tecnologia, Política Nacional de Ciência, Tecnologia e Inovação 
As revistas científicas têm papel fundamental para o desenvolvimento da ciência. A publicação de artigos em revistas científicas permite a avaliação por pares dos resultados das pesquisas, sua divulgação, preservação e mensuração das citações recebidas. Ademais, as revistas publicadas em países emergentes têm relevância para a publicação de artigos com maior interesse nacional ou local, cujo conteúdo não seria atrativo para revistas estrangeiras ${ }^{1}$.

Embora editores e revisores atuem majoritariamente de forma não remunerada, ou realizem a revisão e editoração de artigos como parte de seu trabalho nas instituições aos quais são vinculados, a publicação de revistas científicas requer estrutura adequada e recursos continuados para possibilitar a publicação dos fascículos e volumes com a regularidade e qualidade necessárias. A publicação de uma revista, ainda que somente em formato eletrônico, implica em custos com revisão de idiomas, tradução, normalização, diagramação e editoração eletrônica, além da manutenção da estrutura da secretaria editorial e do sistema de gestão de manuscritos ${ }^{2}$.

Nas revistas de acesso fechado, as despesas são custeadas pelas assinaturas ou pagamentos por acesso aos artigos. Por sua vez, as revistas de acesso aberto podem adotar diferentes modelos de financiamento, desde aqueles em que os autores financiam totalmente a publicação dos artigos, passando por modelos híbridos, nos quais parte dos custos são arcados pelos autores, até modelos nos quais não há cobrança de taxas para submissão ou publicação dos artigos, chamados acesso aberto diamante ou platina ${ }^{3}$.

O Brasil possui uma das experiências de publicação em acesso aberto mais bem sucedidas do mundo, o Programa SciELO. A iniciativa surgiu em 1997, quando a Fundação de Amparo à Pesquisa do Estado de São Paulo (FAPESP) e o Centro Latino-Americano e do Caribe de Informação em Ciências da Saúde (BIREME/OPAS/ OMS) atuaram em parceria para fomentar uma experiência piloto para desenvolvimento da metodologia de publicação online de 10 periódicos brasileiros. No ano seguinte, o SciELO iniciou usas atividades regulares, os objetivos de "desenvolver competência e infraestrutura para indexar e publicar na Internet" e "aumentar a visibilidade, uso e impacto dos periódicos indexados e das pesquisas que publicam". A partir de então, o SciELO expandiu suas atividades e atua na publicação de coleções de revistas de países da América Latina e Caribe, África do Sul, Portugal e Espanha, além de coleções temáticas, como a de Saúde Pública ${ }^{4,5}$.
A Coleção SciELO Brasil, ampliou-se a partir da experiência piloto e, em maio de 2021, contava com 303 periódicos ${ }^{6}$, que constituem um núcleo de periódicos com elevada qualidade (core collection), indexados mediante rigoroso processo de avaliação. A indexação de periódicos na Coleção SciELO Brasil contribui para o aprimoramento dos periódicos, na medida em que aumenta sua visibilidade, tornando-os mais acessíveis aos leitores e atrativos para os autores, e submete os periódicos a critérios de permanência com rigor incremental ${ }^{7}$. Os periódicos têm de buscar, simultaneamente, atender às metas constantes em seus planos de desenvolvimento, e criar estratégias para custear sua implementação e a manutenção de suas atividades regulares. Também o SciELO tem seus custos aumentados com o crescimento da coleção e do número de artigos que os periódicos publicam, com a incorporação de novas tecnologias e com a manutenção de contratos com empresas estrangeiras atrelados ao dólar americano ${ }^{8}$.

Em direção oposta ao desenvolvimento das revistas brasileiras e do Programa SciELO, as perspectivas sobre o financiamento da ciência no Brasil são péssimas. O Ministério da Ciência, Tecnologia e Inovações (MCTI) teve seu orçamento reduzido, o que agravou a situação do Conselho Nacional de Desenvolvimento Científico e Tecnológico $(\mathrm{CNPq})^{9}$. Em 2021 o CNPq recebeu a menor dotação orçamentária do século XXI, sendo o valor $48,5 \%$ menor do que o montante observado no ano 2000 e $64,8 \%$ menor do que o alocado em 2013 (valores atualizados para maio de 2021 segundo IPCA $)^{10}$. O cenário é preocupante pois, o $\mathrm{CNPq}$, juntamente com a Coordenação de Aperfeiçoamento de Pessoal de Nível Superior (CAPES) e as Fundações de Amparo à Pesquisa (FAPs), eram responsáveis por conceder auxílios financeiros às revistas por meio de programas $\mathrm{e}$ editais de fomento. Desde 2013 o financiamento do $\mathrm{CNPq}$ às revistas tem diminuído (Figura 1). Em 2020, o CNPq destinou à Modalidade de Auxílio à Edição e Publicação (AED) apenas R\$ $260.814,87$, o que correspondeu a um apoio de aproximadamente $\mathrm{R} \$ 29.000$ para cada beneficiado (valores atualizados para maio de 2021 segundo IPCA). Os editais, embora sejam fontes importantes de financiamento das revistas, são anuais, no melhor dos cenários, e contemplam apenas uma parcela das revistas e pequena fração de seus custos do período, além do que não garantem segurança financeira às revistas, uma vez que os valores aportados e o número de revistas beneficiadas é variável a cada ano. Em 2021, sequer há previsão de edital para apoio à editora- 


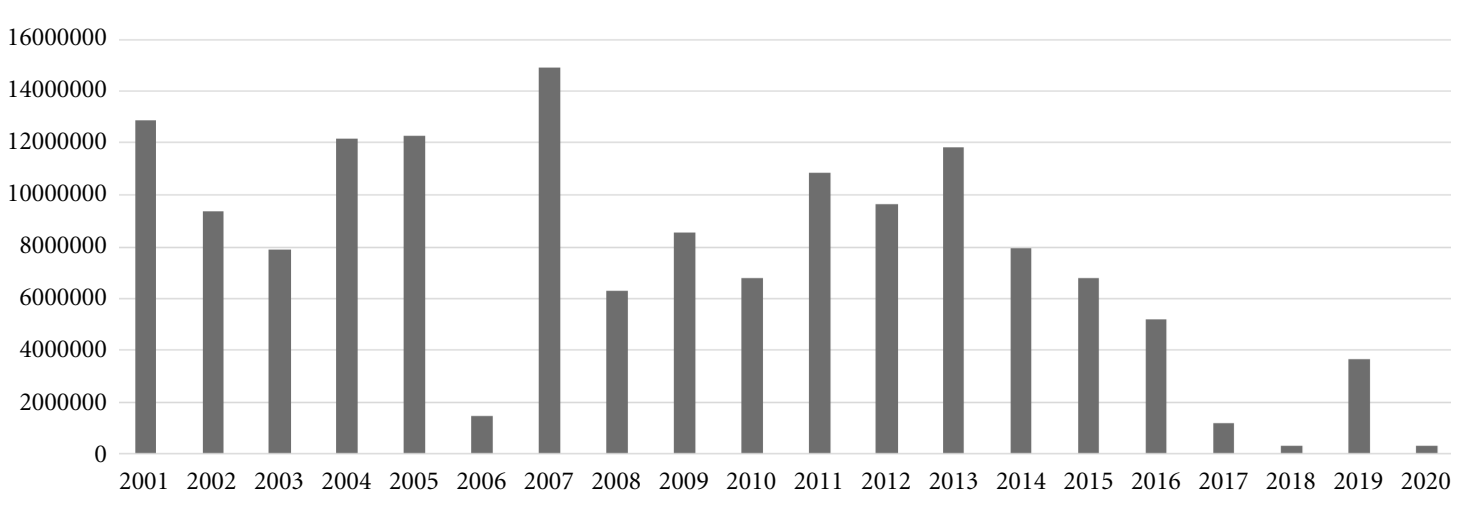

Figura 1. Financiamento do CNPq no Programa Regular de Apoio à Editoração Científica, 2001 a 2020 (em R\$ atualizados pelo IPCA para maio de 2021).

Fonte: $\mathrm{CNPq}^{11}$ (2001-2017), $\mathrm{CNPq}^{12}(2018-2020)$.

ção científica. Assim, no Brasil conjugam-se um baixo financiamento público para a publicação e a ausência de planejamento estratégico com financiamento de longo prazo.

Tal desfinanciamento tem resultado em enorme pressão para as revistas. Algumas delas tiveram prejuízos a suas atividades, como atrasos na publicação, outras passaram a cobrar taxas dos autores, e houve também casos extremos de revistas que encerraram suas atividades. Pesquisa realizada com os editores das revistas indexadas na Coleção SciELO Brasil em 2019/2020 mostrou que $29 \%$ cobravam taxas de processamento de artigos e que $42 \%$ das revistas apresentavam dificuldades de sustentabilidade ${ }^{8}$. Em 2021, é esperado que mais revistas passarão a cobrar taxas dos autores. Neste ano, as duas revistas editadas pela Associação Brasileira de Saúde Coletiva (ABRASCO) aderiram à cobrança de taxas para viabilizar suas existências: Ciência \& Saúde Coletiva para a submissão de artigos e Revista Brasileira de Epidemiologia para publicação.

A situação do SciELO também é preocupante. A partir de 2021 havia a previsão de que o CNPq, a Capes e as FAPs passariam a compartilhar com a FAPESP uma parcela maior do custeio do Programa SciELO. Contudo, no cenário atual de cortes de recursos na área e baixa priorização à ciência, isso não acontecerá. Tais preocupações suscitaram a criação de um Grupo de Trabalho do Comitê Consultivo da Coleção SciELO Brasil para abordar o tema da sustentabilidade e propor estratégias de ação. O grupo recomendou a manutenção do financiamento do SciELO majorita- riamente pela FAPESP e a continuação do apoio pelo $\mathrm{CNPq}$ e pela CAPES. Foi elaborada uma carta, a ser amplamente divulgada, que destaca a importância da garantia de recursos humanos capacitados e de recursos financeiros para a manutenção e o aprimoramento das revistas segundo os requisitos do SciELO. Ela também descreve os principais resultados da pesquisa com editores e informa que, atualmente, os critérios para admissão e permanência de periódicos na Coleção SciELO Brasil são neutros quanto à cobrança de taxas pelas revistas, que devem buscar alternativas de financiamento e que merecem receber apoio das agências de fomento. Todavia, para as revistas que decidirem pela cobrança, o SciELO implantará serviço de apoio para arrecadação e gestão de recursos.

Preocupações semelhantes sobre a sustentabilidade foram levantadas pelos membros do Fórum de Editores das Revistas da Saúde Coletiva que, em conjunto com membros da Diretoria da ABRASCO, buscaram apoio da Sociedade Brasileira para o Progresso da Ciência (SBPC). A SBPC, a Academia Brasileira de Ciências, a Academia Brasileira de Letras e a Academia Nacional de Medicina manifestaram em carta grande preocupação com a falta de financiamento para periódicos científicos brasileiros e com a sustentabilidade das revistas. No mesmo sentido, a ABRASCO e diversas outras entidades estão se mobilizando para tentar sensibilizar CNPq, CAPES e FAPs a respeito da importância do lançamento de editais robustos para apoio a periódicos neste ano de 2021 e nos anos vindouros. 
A posição do Fórum de Editores das Revistas da Saúde Coletiva, alinhada à do Comitê Consultivo da Coleção SciELO Brasil, é de que a publicação de periódicos científicos editados no Brasil é estratégica para o país e parte inerente e fundamental à atividade científica e, como tal, deve ser incentivada por meio de políticas públicas e apoiada com recursos públicos provenientes das instituições mantenedoras e dos editais das agências de fomento. O SciELO, em seus 23 anos de existência, foi financiado como política pública de infraestrutura de pesquisa, e é um programa de colaboração internacional liderado pelo Brasil. As revistas científicas brasileiras e o Programa SciELO são patrimônios nacionais duramente conquistados e centrais para a difusão do conhecimento científico produzido no Brasil, devendo, portanto, ser preservadas. A comunidade científica não hesitará em lutar por sua manutenção, crescimento e fortalecimento.

\section{Colaboradores}

LP Garcia e AF Boing foram responsáveis pela concepção e elaboração do artigo. AF Boing realizou o levantamento dos dados e elaboração da figura. Ambos os autores contribuíram na interpretação dos dados e revisão crítica do artigo, bem como aprovaram a versão final a ser publicada. As opiniões expressas no artigo são dos autores e não necessariamente reconhecidas pelas instituições.

\section{Referências}

1. Meneghini R. Emerging journals. The benefits of and challenges for publishing scientific journals in and by emerging countries. EMBO Rep 2012; 13(2):106-108.

2. Cuenca AMB, Noronha DP, Ueno HM, Kobayashi KM. Periódicos brasileiros de saúde pública - a questão do financiamento. InCID 2011; 2(2):101-110.

3. Taubert N, Hobert A, Fraser N, Jahn N, Iravani E. Open Access - Towards a non-normative and systematic understanding. CoRR 2019; 1910.11568.

4. Packer A, Meneghini R. O SciELO aos 15 anos: raison d'être, avanços e desafios para o futuro. In: Packer A, Cop N, Luccisano A, Ramalho A, Spinak E, organizadores. SciELO - 15 Anos de Acesso Aberto: um estudo analítico sobre Acesso Aberto e comunicação científica. Paris: Unesco; 2014. p. 26-58.

5. Goldbaum M, Antunes JLF, Camargo KR. Relevância dos periódicos de saúde coletiva em informar a pesquisa, a educação, os serviços de saúde e a cidadania. Cien Saude Colet 2021; 26(4):1401-1405.

6. Brasil. Brasil em números [Internet]. [acessado 2021 jun 13]. Disponível em: https://www.scielo.br/?ln$\mathrm{g}=\mathrm{pt}$.

7. Montanari F, Packer AL. Critérios de Seleção de Periódicos para Indexação e Publicação nas Coleções da Rede SciELO. In: Packer A, Cop N, Luccisano A, Ramalho A, Spinak E, organizadores. SciELO - 15 Anos de Acesso Aberto: um estudo analítico sobre Acesso Aberto e comunicação científica. Paris: Unesco; 2014. p. $67-80$.

8. Packer AL. O estado de avanço do Programa SciELO. Reunião Anual SciELO 2020 [Internet]. [acessado 2021 jun 13]. Disponível em: https://scielo.figshare. com/articles/presentation/O_Estado_de_avan_o_ do_Programa_SciELO/13385627.
9. Andrade RO. Ciência à míngua: Sucessivos cortes no orçamento fragilizam a capacidade de financiamento à pesquisa no Brasil. Pesquisa FAPESP [Internet]. 2021 [acessado 2021 jun 13]. Disponível em: https://revistapesquisa.fapesp.br/ciencia-a-mingua.

10. Brasil. Sistema Integrado de Planejamento e Orçamento [Internet]. [acessado 2021 jun 13]. Disponível em: www.siop.planejamento.gov.br.

11. Conselho Nacional de Desenvolvimento Científico e Tecnológico (CNPq). Dados do Painel de Investimentos [Internet]. [acessado 2021 jun 13]. Disponível em: http://estatico.cnpq.br/bi/PagamentoAno/painel-investimentos/painel-dados-painel/index.html.

12. Conselho Nacional de Desenvolvimento Científico e Tecnológico (CNPq). Dados Abertos [Internet]. [acessado 2021 jun 13]. Disponível em: http://dadosabertos.cnpq.br/.

Artigo apresentado em 15/06/2021

Aprovado em 16/06/2021

Versão final apresentada em 18/06/2021

Editores-chefes: Maria Cecília de Souza Minayo, Romeu Gomes, Antônio Augusto Moura da Silva 